\title{
BRYOPHYTE FLORA OF THE BOTANIC GARDEN OF THE UNIVERSITY OF SOPRON (W HUNGARY)
}

\author{
Péter Szücs \\ Eszterházy Károly University, Institute of Biology, Department of Botany and Plant Physiology, \\ H-3300 Eger, Leányka u. 6, Hungary; szucs.peter@uni-eszterhazy.hu
}

Szücs, P. (2017): Bryophyte flora of the Botanic Garden of the University of Sopron (W Hungary). - Studia bot. hung. 48(1): 77-88.

\begin{abstract}
In the course of the research conducted between 2004 and 2008 at the Botanic Garden of the University of Sopron, altogether 78 bryophyte taxa ( 4 liverworts and 74 mosses) were identified. Some Hungarian Red List taxa were detected from the territory: Aphanorrhegma patens, Brachythecium glareosum, Campylium polygamum, Cirriphyllum piliferum, C. tommasinii, Climacium dendroides, Eurhynchium striatum, Orthotrichum obtusifolium, Pottia intermedia, Rhynchostegiella tenella, Rhynchostegium murale, Schistidium elegantulum, and Tortula papillosa.
\end{abstract}

Key words: bryophyte diversity, Hungarian botanic gardens, red list status, semi-natural habitats

\section{INTRODUCTION}

The first significant wave of bryological research conducted in Hungarian botanic gardens was between 1933 and 1968 involving renowned researchers such as László Vajda, Ádám Boros, and József Igmándy. Ádám Boros made visits to Hungarian botanic gardens including Budapest (1933, 1935, 1941, 1942, 1943, 1951), Szarvas (1948, 1960), Vácrátót (1953, 1954), Szeged (1960), and Debrecen (1960) but he also surveyed gardens in neighbouring countries (Kolozsvár, 1941; Pozsony, 1968; Kassa, 1962) and reported sporadic data on the bryoflora of these gardens in his travel diary (BoROs 1915-1971). Majority of these data has never been published, however.

The first description of the bryoflora of the botanic gardens of Vácrátót (VAJDA 1954) and Szigliget (VAJDA 1968) is linked with the name of László Vajda. Bryofloristical data of the botanic garden of Sopron have been published first by József Igmándy (IGMÁNDY 1949).

After a short intermission between 1968 and 1992, the bryofloristical research on Hungarian botanic gardens ran up again. István Galambos (GALAMBOS 1992) published results on the bryoflora of the botanic garden of Zirc.

Several new works were published in succession on already investigated but mostly unexplored botanic gardens, arboretums, and one castle garden: Agostyán 
(Tata) (Szücs 2009), Zirc (Szücs 2013), Eger (SzüCs and PÉnZEsné 2017), Martonvásár (NAGY et al. 2016), Soroksár (NÉMETH and PAPP 2016), Budapest (Buda Arboretum) (Kovács and Rigó 2016).

The present research introduces the bryophyte flora of the Botanic Garden of the University of Sopron, basing on the investigations conducted between 2004 and 2008. Results were also compared with the bryoflora diversity of other Hungarian botanic gardens.

\section{MATERIAL AND METHODS}

In order to characterise the conservation and indicator status of taxa the Hungarian Red List was used (PAPP et al. 2010). The nomenclature of bryophytes follows PAPP et al. (2010). In the case of Hypnum cupressiforme var. lacunosum Hill et al. (2006) was used.

Locality descriptions include data with the following order: habitat, GPS coordinates, date of collection. The identifiers of the quadrates, according to the Central European Flora Mapping System, were indicated in square brackets (KirÁly 2003).

Collected individuals are stored at the Cryptogamic Herbarium of the Department of Botany and Plant Physiology at the Eszterházy Károly University, Eger (EGR).

\section{Study area}

The Botanical Garden of the University of Sopron is located in Győr-MosonSopron County, in the territory of the local administrative unit of Sopron, in the eastern foothills of the Sopron Hills. The extension of the territory is 17 hectares (Fig. 1). From the phytogeographical point of view the Sopron Hills are situated in the border region of the Alpine (Alpicum) and the Pannonian (Pannonicum) floristic provinces. The mean annual temperature varies between $8-9{ }^{\circ} \mathrm{C}$, and the annual precipitation is usually between 650-900 mm (KIRÁLY 2004, SZMORAD 2011). The soils of the botanical garden were developed on loess, marl, and marllike sediments, and the soil texture is loam and clayey-loam. The average soil pH is slightly acidic in the territory (FoLCZ and BöRCsöK 2015).

The garden contains 2691 woody plant taxa, 3691 herbaceous taxa, and 171 mushroom species. Among the taxa there are $16 \mathrm{in} \mathrm{situ} \mathrm{and} 135 \mathrm{ex} \mathrm{situ} \mathrm{protected}$ plant species, as well as 2 protected mushroom species (Kocsó 2008, FolCz and BörcsöK 2015). 


\section{Site details}

Collection of the specimens was carried out in Györ-Moson-Sopron County, in the territory of the local administrative unit of Sopron. Each collection point belongs to 8365.1 quadrant.

1. Rockery, stone wall, mown lawn, and roadside, bare soil surface, $47.680033^{\circ} \mathrm{N}, 16.572674^{\circ} \mathrm{E}$ (04.02.2004, 25.06.2004, 26.06.2004, 27.06.2004, 24.09.2005, 12.04.2006, 24.09.2008)

2. Stone building, mown lawn, and roadside, woody vegetation, bare soil surface, $47.679451^{\circ} \mathrm{N}$, $16.573767^{\circ} \mathrm{E}(20.01 .2004,04.02 .2004,25.06 .2004,24.09 .2005,12.04 .2006)$

3. Mown lawn, roadside, woody vegetation, bare soil surface, embankment of road, $47.678624^{\circ} \mathrm{N}$, $16.574742^{\circ} \mathrm{E}(20.01 .2004,04.02 .2004,27.06 .2004,24.09 .2005,12.04 .2006)$

4. Embankment of road, mown lawn, drainage ditch, $47.680744^{\circ} \mathrm{N}, 16.573269^{\circ} \mathrm{E}(04.02 .2004$, 25.06.2004, 26.06.2004, 12.04.2006)

5. Stone wall, embankment of road, drainage ditch, $47.679634^{\circ} \mathrm{N}, 16.575648^{\circ} \mathrm{E}(20.01 .2004$, 04.02.2004, 25.06.2004, 26.06.2004, 27.06.2004)

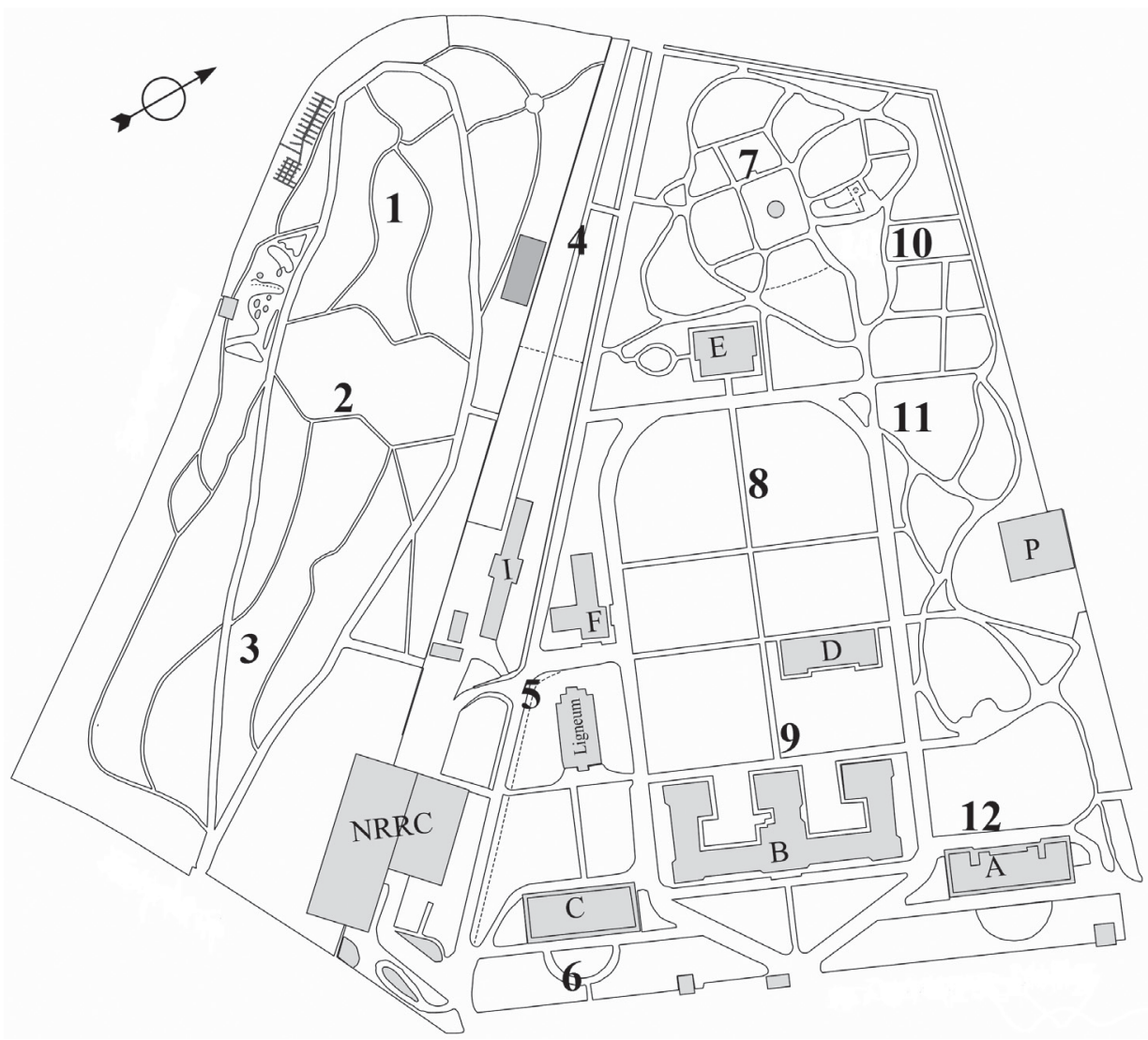

Fig. 1. Map of the botanical garden of the University of Sopron with the sampling sites (M $1: 3000$ ). 
6. Rockery and mown lawn, $47.679232^{\circ} \mathrm{N}, 16.577297^{\circ} \mathrm{E}$ (04.02.2004, 25.06.2004, 26.06.2004, 27.06.2004, 24.09.2005, 12.04.2006)

7. Mown lawn, roadside, woody vegetation, $47.681264^{\circ} \mathrm{N}, 16.573834^{\circ} \mathrm{E}(04.02 .2004,25.06 .2004$, 26.06.2004, 27.06.2004, 12.04.2006)

8. Mown lawn, roadside, bare soil surface, $47.680681^{\circ} \mathrm{N}, 16.575495^{\circ} \mathrm{E}(20.01 .2004,04.02 .2004$, 25.06.2004, 24.09.2015, 12.04.2006)

9. Mown lawn, embankment of road, bare soil surface, concrete, $47.680388^{\circ} \mathrm{N}, 16.576685^{\circ} \mathrm{E}$ (20.01.2004, 04.02.2004, 25.06.2004, 26.06.2004, 27.06.2004)

10. Forest area, roadside, woody vegetation, $47.681751^{\circ} \mathrm{N}, 16.574057^{\circ} \mathrm{E}(04.02 .2004,25.06 .2004$, 26.06.2004)

11. Forest area, roadside, woody vegetation, $47.681578^{\circ} \mathrm{N}, 16.575339^{\circ} \mathrm{E}(04.02 .2004,26.06 .2004$, 27.06.2004)

12. Bare soil surface, anthropogenic habitat, $47.681097^{\circ} \mathrm{N}, 16.577539^{\circ} \mathrm{E}(20.01 .2004,04.02 .2004$, 25.06.2004, 26.06.2004, 27.06.2004, 24.09.2005, 12.04.2006)

\section{RESULTS}

Number of taxa, conservation status, indicator species

According to the present investigations 78 bryophytes were collected in the Botanic Garden of the University of Sopron, including 4 liverworts and 74 mosses. Besides the common and frequent taxa, mosses which are still not threatened, but need attention (LC-att) according to the Hungarian Bryophyte Red List, can be found (e.g. Brachythecium albicans, Cirriphyllum tommasinii, Climacium dendroides, Lophocolea bidentata, Pottia intermedia, Rhynchostegium murale and Tortula papillosa).

Near threatened species were as follows: Aphanorrhegma patens, Brachythecium glareosum, Campylium polygamum, Cirriphyllum piliferum, Orthotrichum obtusifolium, and Rhynchostegiella tenella.

Indicator species which by their mere presence denote the greater level of conservation value of the habitat, also occur in the garden like Lophocolea bidentata, Campylium polygamum, Cirriphyllum piliferum, Cirriphyllum tommasinii, Orthotrichum cupulatum, Pottia intermedia, and Rhynchostegiella tenella.

\section{List of species}

Numbers refer to sites listed above. The substrates given after a semicolon refer to all listed sites.

\section{Hepaticeae}

Lophocolea bidentata (L.) Dumort. - 4; on shady soil Marchantia polymorpha L. - 12; on artificial stone 
Pellia endiviifolia (Dicks.) Dumort. - 5; on shady, wet soil

Radula complanata (L.) Dumort. - 8; bark of Populus xeuramericana

Musci

Amblystegium serpens (Hedw.) Schimp. - 1, 2: on bark of Acer platanoides, 3, 7: on bark of Aesculus hippocastanum, 8: on bark of Aesculus hippocastanum; on soil

Amblystegium tenax (Hedw.) C. E. O. Jensen - 5; on shaded, wet stone

Aphanorrhegma patens (Hedw.) Lindb. - 6; on shady, wet, clayey soil

Atrichum undulatum (Hedw.) P. Beauv. - 3; on soil

Barbula convoluta Hedw. - 1, 5, 6; on exposed soil

Barbula unguiculata Hedw. - 1, 2, 3, 5, 6, 8, 12; on exposed soil

Brachythecium albicans (Hedw.) Schimp. 1, 5, 6; on exposed soil

Brachythecium glareosum (Spruce) Schimp. - 5; on concrete and stone

Brachythecium rutabulum (Hedw.) Schimp. - 2, 3, 6, 7, 8, 9; on soil

Brachythecium salebrosum (F. Weber et D. Mohr) Schimp. - 1: on bark of Betula pendula, 8; on bark of Aesculus hippocastanum

Brachythecium velutinum (Hedw.) Schimp. - 1, 3, 7; on shady soil

Bryum argenteum Hedw. - 1, 5, 6, 12; on exposed soil

Bryum bicolor Dicks. - 1; on soil

Bryum caespiticium Hedw. - 1, 5, 12; on exposed soil

Bryum capillare Hedw. - 6, 7; on soil

Bryum moravicum Podp. - 5; on bark of Robinia pseudoacacia; 6; on bark of Tilia tomentosa

Calliergonella cuspidata (Hedw.) Loeske - 7, 8; on wet soil

Campylium calcareum Crundw. et Nyholm - 6; on shady limestone

Campylium chrysophyllum (Brid.) Lange - 9; on soil

Campylium polygamum (Schimp.) C. E. O. Jensen - 8; on wet soil

Ceratodon purpureus (Hedw.) Brid. - 1: on stone and exposed soil; 4, 5: on stone and concrete, 12 ; on artificial stone

Cirriphyllum piliferum (Hedw.) Grout - 3, 7, 8; on soil

Cirriphyllum tommasinii (Boulay) Grout - 5; on shaded stone

Climacium dendroides (Hedw.) F. Weber et D. Mohr - 7, 8; on shaded soil

Cratoneuron filicinum (Hedw.) Spruce - 6; on soil

Dicranella heteromalla (Hedw.) Schimp. - 11; on soil

Dicranella varia (Hedw.) Schimp. - 8; on soil

Didymodon vinealis (Brid.) R. H. Zander - 9; on soil

Eurhynchium angustirete (Broth.) T. J. Kop. - 3; on soil

Eurhynchium hians (Hedw.) Sande Lac. - 2, 3, 4, 6, 7, 8, 9, 11; on soil 
Eurhynchium striatum (Hedw.) Schimp. - 3; on soil

Fissidens bryoides Hedw. - 7; on soil

Fissidens taxifolius Hedw. - 7, 8, 9; on bare soil

Funaria hygrometrica Hedw. - 2, 12; on soil

Grimmia pulvinata (Hedw.) Sm. - 1: on stone, 5; on stone and concrete

Homalothecium lutescens (Hedw.) H. Rob. - 1, 9; on exposed soil

Homalothecium philippeanum (Spruce) Schimp. - 1; on limestone

Hylocomium splendens (Hedw.) Schimp. - 7; on shaded soil

Hypnum cupressiforme Hedw. - 1, 2, 3, 4, 5, 6, 7, 8, 9, 10, 11, 12; soil, stone, concrete, bark of trees

Hypnum cupressiforme var. lacunosum Brid. - 5; on exposed stone

Leskea polycarpa Ehrh. ex Hedw. - 4, 5, 8; on bark of Populus

Orthotrichum affine Schrad. ex Brid. - 5, 8; on bark of Populus

Orthotrichum anomalum Hedw. - 1; on exposed limestone

Orthotrichum cupulatum Brid. - 5; on exposed stone

Orthotrichum diaphanum Schrad. ex Brid. - 6: on bark of Tilia tomentosa, stone;

8; on bark of Populus

Orthotrichum obtusifolium Brid. - 5; on bark of Fraxinus angustifolia

Orthotrichum stramineum Hornsch. ex Brid. - 5: on bark of Fraxinus; 10; on bark of Acer pseudoplatanus

Phascum cuspidatum Hedw. - 1, 2, 5; on bare soil

Physcomitrium pyriforme (Hedw.) Brid. - 5; on wet, shaded soil

Plagiomnium affine (Blandow) T. J. Kop. - 7, 8; on soil

Plagiomnium cuspidatum (Hedw.) T. J. Kop. - 3, 5, 8; on soil

Plagiomnium rostratum T. J. Kop. - 2, 4, 5, 8; on soil

Plagiomnium undulatum (Hedw.) T. J. Kop. - 3, 4, 5, 8, 9; on soil

Plagiothecium cavifolium (Brid.) Z. Iwats. - 5; on soil

Platygyrium repens (Brid.) Schimp. - 2, 7; on bark of Quercus

Pohlia melanodon (Brid.) A. J. Shaw - 8; on soil

Pottia intermedia (Turner) Fürnr. - 1; on soil

Pottia lanceolata (Hedw.) Müll. Hal. - 1; on wet, shady soil

Pseudocrossidium hornschuchianum (Schultz) R. H. Zander - 1; on limestone

Pylaisia polyantha (Hedw.) Schimp. - 5: on bark of Fraxinus, 6; on bark of Tilia tomentosa

Rhynchostegiella tenella (Dicks.) Limpr. - 5; on shaded, wet stone

Rhynchostegium murale (Hedw.) Schimp. - 5; on concrete

Rhytidiadelphus squarrosus (Hedw.) Warnst. - 7; on soil

Rhytidiadelphus triquetrus (Hedw.) Warnst. - 7, 8; on soil

Schistidium crassipilum H. H. Blom - 6; on muscovite stone (ERZBERGER and SCHRÖDER 2008) 
Schistidium elegantulum H. H. Blom - 9; on concrete (ERZBERger and SCHRÖDER 2008)

Scleropodium purum (Hedw.) Limpr. - 3, 4, 7, 8; on soil

Thuidium abietinum (Hedw.) Schimp. - 8, 9; on soil

Thuidium delicatulum (Hedw.) Schimp. - 7, 8; on soil

Thuidium philibertii Limpr. - 7, 8; on soil

Thuidium tamariscinum (Hedw.) Schimp. - 7; on soil

Tortula muralis L. ex Hedw. - 1, 5, 6; on stone, 2, 3; on bitumen

Tortula papillosa Wilson - 5; on bark of Fraxinus

Tortula ruralis (Hedw.) P. Gaertn., B. Mey. et Scherb. - 1: on soil and stone; 5; on stone

\section{DISCUSSION}

Some common bryophytes of the botanic garden of Sopron, including $A m$ blystegium serpens, Barbula unguiculata, Brachythecium rutabulum, B. salebrosum, Eurhynchium hians, Bryum argenteum, Ceratodon purpureus, Hypnum cupressiforme, Leskea polycarpa, Orthotrichum anomalum, O. diaphanum, Radula complanata, Tortula muralis, and Tortula ruralis occur also in almost all investigated Hungarian botanic gardens and arboretums (Table 1). $62.8 \%$ of the species identified in the Botanic Garden of the University of Sopron can also be found in the Botanic Garden of Vácrátót. The results for other gardens and arboretums are as follows: Agostyán 40.0\%, Eger 34.6\%, Budapest 39.7\%, Martonvásár 48.7\%, Soroksár 50.5\%, Szigliget 37.2\%, and Zirc 52.6\%.

Some less common mosses (Amblystegium tenax, Fissidens bryoides, Rhynchostegiella tenella) were detected only in Sopron. The values show that the bryophyte flora of the Botanic Garden of the University of Sopron closely resembles the flora of some other investigated gardens (Zirc, Martonvásár, Soroksár) and the arboretum of Vácrátót, despite the long distance of the latter from Sopron. The high similarity can primarily be explained by the high number of species and the presence of common and frequent bryophytes.

The differences of the bryophyte flora of various gardens can mainly be explained by different habitats and natural conditions, various dimensions, different ages and level of bryophyte investigations (Fig. 2, Table 1).

The low proportion of liverworts (Fig. 2) in the investigated area is similar to the majority of other Hungarian botanic gardens and arboretums. However, this phenomenon is also very characteristic for many European cities and settlements (Szücs et al. 2017). 
Table 1. Occurrence of species in the Sopron Botanic Garden, in other Hungarian botanic gardens, arboretums and manor parks and percentage of similarity of bryophyte flora with that of Sopron Botanic Garden. Comparative data were obtained from the following sources: Agostyán (SzÜCs 2009), Eger (SzÜCS and PÉNZESNÉ-KóNYA 2017), Budapest (Kovács and Rigó 2016), Martonvásár (NAGY et al. 2016), Soroksár (NÉmeth and Papp 2016), Szigliget (Vajda 1968), Vácrátót (Vajda 1954), Zirc (Galambos 1992, SzÜCs 2013).

\section{Botanic gardens}

Taxon name

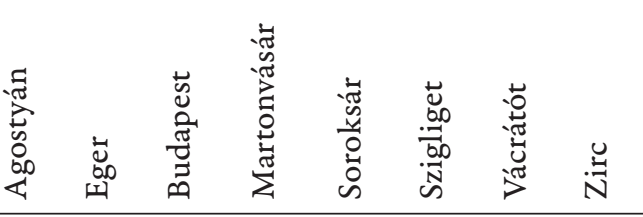

\begin{tabular}{|c|c|c|c|c|c|c|c|c|}
\hline \multicolumn{9}{|c|}{ Hepaticeae } \\
\hline Lophocolea bidentata & - & - & - & - & - & - & + & + \\
\hline Marchantia polymorpha & - & - & + & + & + & - & + & + \\
\hline Pellia endiviifolia & + & - & + & - & - & - & - & - \\
\hline Radula complanata & + & - & + & + & + & + & + & + \\
\hline \multicolumn{9}{|c|}{ Musci } \\
\hline Amblystegium serpens & + & + & + & + & + & + & + & + \\
\hline Amblystegium tenax & - & - & - & - & - & - & - & - \\
\hline Aphanorrhegma patens & - & - & - & + & - & - & - & - \\
\hline Atrichum undulatum & + & - & - & & - & + & - & + \\
\hline Barbula convoluta & - & - & + & + & - & - & - & + \\
\hline Barbula unguiculata & + & + & + & + & + & + & + & + \\
\hline Brachythecium albicans & + & - & - & - & + & - & + & - \\
\hline Brachythecium glareosum & - & - & - & - & - & - & + & + \\
\hline Brachythecium rutabulum & + & + & + & + & + & + & + & + \\
\hline Brachythecium salebrosum & + & + & + & + & + & + & + & - \\
\hline Brachythecium velutinum & + & - & - & - & + & - & + & + \\
\hline Bryum argenteum & + & + & + & + & + & + & + & - \\
\hline Bryum bicolor & - & - & - & - & + & - & - & - \\
\hline Bryum caespiticium & - & + & - & + & + & - & + & - \\
\hline Bryum capillare & - & - & + & + & + & - & + & + \\
\hline Bryum moravicum & - & - & + & + & + & - & - & + \\
\hline Calliergonella cuspidata & - & + & + & + & + & + & + & - \\
\hline Campylium calcareum & - & - & - & - & + & - & - & - \\
\hline
\end{tabular}


Table 1. (continued).

\section{Botanic gardens}

Taxon name

\begin{tabular}{|c|c|c|c|c|c|c|c|c|}
\hline 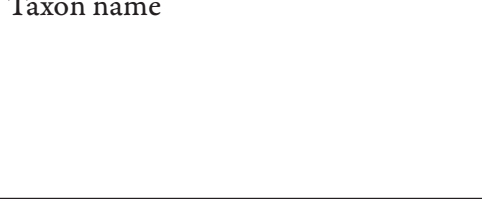 & 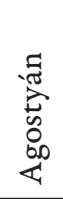 & $\begin{array}{l}\overrightarrow{\mathrm{D}} \\
\text { 品 }\end{array}$ & 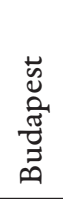 & 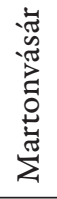 & 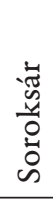 & 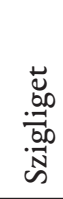 & 蒡 & $\stackrel{\mathscr{N}}{\mathrm{N}}$ \\
\hline Campylium chrysophyllum & - & - & - & - & - & - & + & + \\
\hline Campylium polygamum & + & - & - & - & - & - & - & - \\
\hline Ceratodon purpureus & + & + & + & + & + & + & + & - \\
\hline Cirriphyllum piliferum & - & - & - & - & - & + & - & + \\
\hline Cirriphyllum tommasinii & + & - & - & - & - & - & + & + \\
\hline Climacium dendroides & - & + & - & - & - & - & - & - \\
\hline Cratoneuron filicinum & + & - & - & - & - & - & + & + \\
\hline Dicranella heteromalla & + & - & - & - & - & - & - & - \\
\hline Dicranella varia & - & - & + & - & - & - & - & + \\
\hline Didymodon vinealis & - & - & - & - & - & + & - & - \\
\hline Eurbynchium angustirete & + & - & - & - & - & - & + & - \\
\hline Eurbynchium hians & + & + & + & + & + & + & + & + \\
\hline Eurhynchium striatum & + & - & - & - & - & - & - & - \\
\hline Fissidens bryoides & - & - & - & - & - & - & - & - \\
\hline Fissidens taxifolius & + & + & + & + & - & - & + & + \\
\hline Funaria hygrometrica & - & - & - & + & + & + & + & + \\
\hline Grimmia pulvinata & + & - & + & + & + & + & + & - \\
\hline Homalothecium lutescens & + & + & - & + & + & - & + & + \\
\hline Homalothecium philippeanum & - & + & - & - & - & - & + & + \\
\hline Hylocomium splendens & - & + & - & - & - & - & + & - \\
\hline Hypnum cupressiforme & + & + & + & + & + & + & + & + \\
\hline Hypnum cupressiforme var. lacunosum & + & - & - & - & - & - & - & - \\
\hline Leskea polycarpa & + & - & + & + & + & + & + & + \\
\hline Orthotrichum affine & - & - & + & + & + & - & + & + \\
\hline Orthotrichum anomalum & - & + & + & + & + & + & + & + \\
\hline Orthotrichum cupulatum & - & + & + & + & + & - & - & - \\
\hline Orthotrichum diaphanum & - & + & + & + & + & + & + & + \\
\hline Orthotrichum obtusifolium & - & - & + & + & + & - & + & + \\
\hline Orthotrichum stramineum & - & - & - & - & + & - & - & - \\
\hline
\end{tabular}


Table 1. (continued).

\section{Botanic gardens}

Taxon name

\begin{tabular}{|c|c|c|c|c|c|c|c|c|}
\hline & 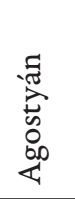 & 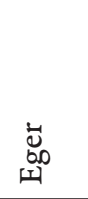 & 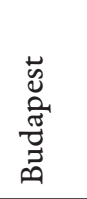 & 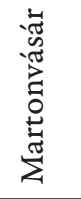 & 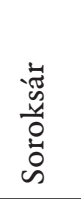 & 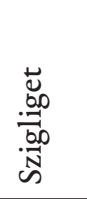 & 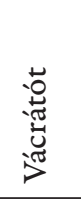 & $\stackrel{\mathscr{N}}{\mathrm{N}}$ \\
\hline Phascum cuspidatum & - & - & - & + & - & + & + & + \\
\hline Physcomitrium pyriforme & - & - & - & + & + & - & + & + \\
\hline Plagiomnium affine & + & - & - & + & - & + & - & - \\
\hline Plagiomnium cuspidatum & - & + & + & + & + & - & + & + \\
\hline Plagiomnium rostratum & - & - & - & - & - & - & - & + \\
\hline Plagiomnium undulatum & + & + & - & + & - & + & + & + \\
\hline Plagiothecium cavifolium & + & - & - & - & - & - & - & - \\
\hline Platygyrium repens & - & + & - & + & - & - & - & + \\
\hline Poblia melanodon & - & - & - & + & - & - & - & - \\
\hline Pottia intermedia & - & - & - & - & - & - & + & - \\
\hline Pottia lanceolata & - & + & - & - & + & - & + & - \\
\hline Pseudocrossidium hornschuchianum & - & - & + & - & + & - & + & - \\
\hline Pylaisia polyantha & - & - & + & - & + & + & + & + \\
\hline Rhynchostegiella tenella & - & - & - & - & - & - & - & - \\
\hline Rhynchostegium murale & - & - & - & - & - & + & - & + \\
\hline Rhytidiadelphus squarrosus & - & + & - & - & - & + & - & - \\
\hline Rhytidiadelphus triquetrus & - & - & - & - & - & - & + & + \\
\hline Schistidium crassipilum & + & + & + & + & + & - & - & + \\
\hline Schistidium elegantulum & - & - & - & - & - & - & - & - \\
\hline Scleropodium purum & + & + & - & - & + & + & + & + \\
\hline Thuidium abietinum & - & - & - & - & + & - & + & - \\
\hline Thuidium delicatulum & - & - & - & - & - & - & + & - \\
\hline Thuidium philibertii & + & + & - & + & - & + & + & - \\
\hline Thuidium tamariscinum & - & - & - & - & - & - & + & - \\
\hline Tortula muralis & - & + & + & + & + & + & + & + \\
\hline Tortula papillosa & - & - & + & + & + & + & + & - \\
\hline Tortula ruralis & + & - & + & + & + & + & + & + \\
\hline $\begin{array}{l}\text { Percentage of similitude with Botanic } \\
\text { Garden of Sopron (\%) }\end{array}$ & 40.0 & 34.6 & 39.7 & 48.7 & 50.5 & 37.2 & 62.8 & 52.6 \\
\hline
\end{tabular}




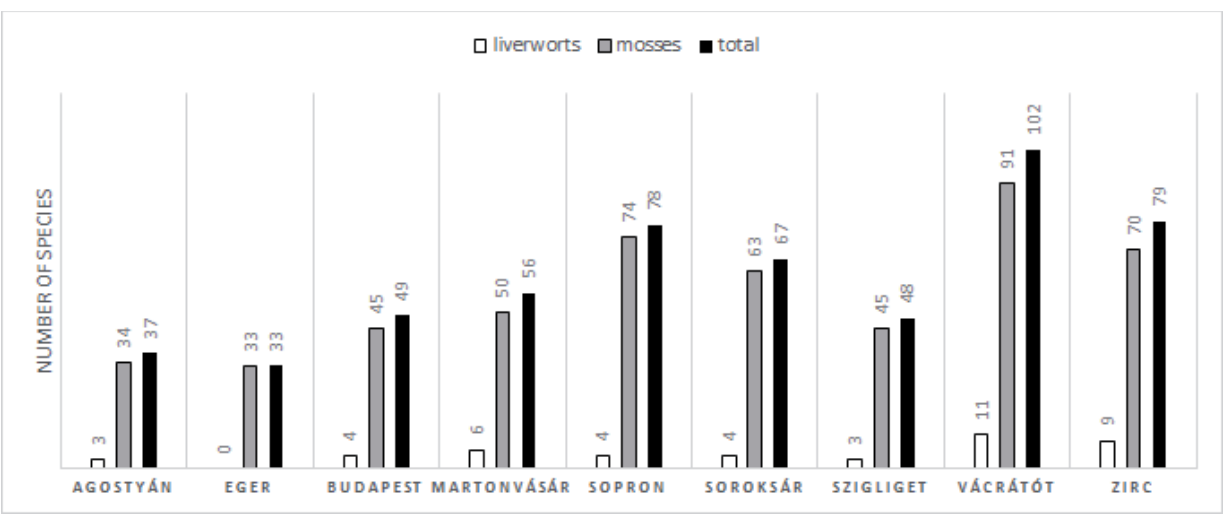

Fig. 2. The number of species of Hungarian botanic gardens, arboretums, and manor parks. Comparative data were obtained from the following sources: Agostyán (Szücs 2009), Eger (Szücs and PÉnzesné-Kónya 2017), Budapest (KovÁcs and Rigó 2016), Martonvásár (NAgy et al. 2016), Sopron (this work), Soroksár (NÉmETH and PAPP 2016), Szigliget (VAJdA 1968), Vácrátót (VAjda 1954), Zirc (Galambos 1992, Szücs 2013).

Acknowledgement - The author would like to express his gratitude to Peter Erzberger for his help in sample analysis. This research was supported by the grant EFOP-3.6.1-16-2016-00001 (“Complex improvement of research capacities and services at Eszterházy Károly University”).

Összefoglaló: A 2004. és 2008. között végzett kutatás alapján a Sopron Egyetem Botanikus Kertjének területéről összesen 78 mohafaj ismert, melyből 4 a májmohákhoz 74 pedig a lombosmohákhoz tartozik. Figyelemre méltó mohafajok a hazai vörös lista alapján: Aphanorrhegma patens, Brachythecium glareosum, Campylium polygamum, Cirriphyllum piliferum, Cirriphyllum tommasinii, Climacium dendroides, Eurhynchium striatum, Orthotrichum obtusifolium, Pottia intermedia, Rhynchostegiella tenella, Rhynchostegium murale, Schistidium elegantulum és Tortula papillosa.

\section{REFERENCES}

Boros, Á. (1915-1971): Florisztikai jegyzetek. - Hungarian Natural History Museum, Budapest. ERzBerger, P. and Schröder, W. (2008): The genus Schistidium (Grimmiaceae, Musci) in Hungary. - Studia bot. hung. 39: 27-88.

FolCZ, Á. and BöRCsöK, Z. (2015): Macrofungi in the Botanical Garden of the University of West Hungary, Sopron - Acta Silvatica \& Lignaria Hungarica 1 1(2): 111-122.

Galambos, I. (1992): A Zirci Arborétum mohaflórája. - Folia Mus. hist.-nat. Bakony. 11:29-35.

Hill, M. O., Bell, N., Bruggeman-Nannaenga, M. A., Brugues, M., Cano, M. J., Enroth, J., Flatberg, K. I., Frahm, J. P., Gallego, M. T., Gariletti, R., Guerra, J., Hedenäs, L., Holyoak, D. T., Hyvönen, J., Ignatov, M. S., Lara, F., Mazimpaka, V., Muñoz, J. and SÖDERSTRÖM, L. (2006): An annotated checklist of the mosses of Europe and Macaronesia. - J. Bryol. 28: 198-267.

IGMÁNDY, J. (1949): Adatok Sopron mohaflórájához. - Erd. kísérl. 49(1-4): 164-167. 
KirÁLy, G. (2003): A magyarországi flóratérképezés módszertani alapjai. - Flora Pannonica 1: $3-20$.

KIrÁLY, G. (2004). A Soproni-hegység edényes flórája. (Die Gefäßpflanzenflora des Ödenburger Gebirges). - Flora Pannonica 2(1): 7-12.

Kocsó, M. (2008): Az erdészeti felsöoktatás botanikus kertje (Botanical Garden of the foresters higher education). - In: Albert, L. (ed.): Az erdészeti felsőoktatás 200 éve. Nyugat-magyarországi Egyetem Erdömérnöki Kar, Sopron, pp.145-165.

Kovács, A. and Rigó, A. (2016): A Budai Arborétum moha-és zuzmóflórája. - manuscript, Corvinus University of Budapest, Budapest, $50 \mathrm{pp}$.

NAGY, Z., MAJLÁth, I., MolnáR, M. and ERZBerger, P. (2016): A martonvásári kastélypark mohaflórája. - Kitaibelia 21(2): 198-206.

NÉMeth, Cs. and PAPP, B. (2016): Mohák a Soroksári Botanikus Kertben. - In: HöHN, M. and PAPP, V. (eds): Biodiverzitás a Soroksári Botanikus Kertben. Magyar Biodiverzitás-kutató Társaság and SZIE Kertészettudományi Kar, Soroksári Botanikus Kert, Budapest, pp. 111-149.

Papp, B., Erzberger, P., Ódor, P., Hock, Zs., Szövényi, P., SZurdoki, E. and Tóth, Z. (2010): Updated checklist and redlist of Hungarian bryophytes. - Studia bot. hung. 41: 31-59.

Szmorad, F. (2011): The Riparian Alder Forests of the Sopron Hills. - Acta Silvatica \& Lignaria Hungarica 7: 109-124.

Szücs, P. (2009): Mohaadatok az Agostyáni Arborétumból. - Komárom-Esztergom Megyei Múz. Közlem. 15: 159-164.

SzÜcs, P. (2013): Kiegészítések a Zirci Arborétum mohaflórájához. - Folia Mus. hist.-nat. Bakony. 30: 47-54.

SzÜCs, P. and PÉNZesné KónYA, E. (2017): Mohaadatok az Eszterházy Károly Főiskola Botanikus Kertjéből (Eger). - Acta Acad. Paed. Agr., Sect. Biol. 41 (in press).

SzÜcs, P., PÉnzes-Kónya, E. and Hofmann, T. (2017): The bryophyte flora of the village of Almásfüzitő, a former industrial settlement in NW-Hungary. - Cryptogamie, Bryol. 38(2): 153-170.

VAJDA, L. (1954): A Vácrátóti Botanikai Kutató Intézet Természetvédelmi Parkjának mohái. (Die Moose im Naturschutzparke des Botanischen Forschungsinstitutes von Vácrátót.) - Bot. Közlem. 45: 63-66.

VAJDA, L. (1968): A Szigligeti Arborétum mohái. (Die Moose des Arboretums Szigliget.) - A Veszprém megy. Múz. Közlem. 7: 237-240.

(submitted: 02.04.2017, accepted: 30.04 .2017 ) 